\title{
Radioiodine therapy increases the risk of cerebrovascular events in hyperthyroid and euthyroid patients
}

\author{
Jeppe Lerche la Cour, Lars Thorbjoern Jensen ${ }^{1}$, Anders Vej-Hansen ${ }^{2}$ and \\ Birte Nygaard ${ }^{3}$
}

Department of Clinical Physiology, Nuclear Medicine and PET, Rigshospitalet, University of Copenhagen, Nodre Ringvej 57, 2600 Glostrup, Denmark, 'Department of Clinical Physiology and Nuclear Medicine, University Hospital of Herlev, Herlev Ringvej 75, 2730 Herlev, Denmark, ${ }^{2}$ Primary Health Care, Region Zealand, Ågade 10, 4700 Næstved, Denmark and ${ }^{3}$ Section of Endocrinology, Department of Medicine, University Hospital of Herlev, Herlev Ringvej 75, 2730 Herlev, Denmark
Correspondence should be addressed to J L la Cour Email jeppe.lerche.la.cour.01@ regionh.dk or jeppelerche@hotmail.com

\begin{abstract}
Background and objective: Hyperthyroid patients treated with radioiodine have increased morbidity and mortality from cerebrovascular events. This risk has until now has been attributed to the hyperthyroidism. However, radioiodine therapy of benign thyroid diseases exposes the carotid arteries to radiation and is capable of inducing atherosclerosis. The objective of the study was to elucidate whether ionizing radiation from radioiodine might contribute to cerebrovascular morbidity. Methods: In a retrospective register cohort study, 4000 hyperthyroid and 1022 euthyroid goitre patients treated with radioiodine between 1975 and 2008 were matched 1:4 on age and sex with random controls. The cohort was followed from the date of treatment until hospitalization due to cerebrovascular event, death, 20 years of follow-up or March 2013. Data were analyzed in competing risk models adjusting for age, sex, Charlson's comorbidity score, atrial fibrillation and previous cerebrovascular events.

Results: Mean follow-up time was 11.5 years, mean age 61 years, with a total number of 3228 events. Comparing all radioiodine-treated patients with controls, the fully adjusted model showed increased risk of cerebrovascular events among all treated patients, hazard ratio (HR) 1.18 (95\% Cl 1.09-1.29). The risk was increased among hyperthyroid (HR 1.17; 95\% Cl 1.07-1.28) as well as euthyroid patients (HR 1.21; 95\% Cl 1.02-1.44).

Conclusions: We report an increased risk of cerebrovascular events in hyperthyroid as well as euthyroid patients treated with radioiodine for benign thyroid disorders. That these patient groups have similar risks suggests the possibility that radiation from radioiodine contributes to cerebrovascular morbidity via acceleration or initiation of atherosclerosis.
\end{abstract}

\section{Introduction}

It is recognized that ionizing radiation induces or accelerates the development of atherosclerosis when arteries are exposed. An increased risk of atherosclerotic events can be seen already from the first years after exposure (1). As the thyroid and carotid arteries are in close contact, the carotid arteries are very likely to be exposed to radiation during treatment with radioactive iodine (RAI) for benign thyroid disorders (2). This raises the concern that these patients might have an increased risk of atherosclerotic cerebrovascular events, i.e. stroke.

Indeed, an increased risk of mortality from cerebrovascular events has been shown in studies of hyperthyroid patients treated with RAI $(3,4)$ (hyperthyroid defined as a thyroid-stimulating hormone (TSH) $<0.01$ and elevated free thyroid hormones (tri-iodothyronine $\left(\mathrm{T}_{3}\right)$ and/or thyroxine $\left(\mathrm{T}_{4}\right)$ ). This risk has been attributed to the 
hyperthyroidism not RAI, as hyperthyroidism has many established vascular effects such as hypercoagulopathy (5), increased risk of atrial fibrillation (AF), increased left ventricular ejection fraction and heart failure (6). The theory that it is the hyperthyroidism per se that holds the risk is supported by Sheu et al. (7), who demonstrated an increased risk of stroke among hyperthyroid treated with anti-thyroid medicine. However, other studies of hyperthyroid patients treated with RAI, surgery and/or medical treatment have described a relationship between hyperthyroidism and cardiac - but not cerebrovascular mortality or morbidity $(8,9,10,11,12)$. Unfortunately, data on cerebrovascular events in euthyroid goitre patients treated with RAI for symptoms such as compression of trachea or dysphagia are absent. Data on this type of patients could, perhaps, have given us an indication on whether it is hyperthyroidism per se or RAI that leads to the increased risk of cerebrovascular events in RAI-treated hyperthyroid patients.

As a cerebrovascular event is serious, we find this possible adverse effect of RAI important to examine. Should an excess risk exist, it would be an important factor to take into account when making the decision to treat with either thyroidectomy or RAI. Another important aspect is that the patients should be properly informed on effects and possible serious side effects before accepting any of the treatments.

\section{Objectives}

Therefore we wanted to elucidate to what extent RAI might be a factor in the increased risk of cerebrovascular events among RAI-treated hyperthyroid patients. Thus, we aimed to investigate whether there was a difference in the hazard ratio (HR) of hospitalization from cerebrovascular events between RAI-treated patients, both hyperthyroid and euthyroid, compared with random thyroid healthy controls.

\section{Subjects and methods}

\section{Study population}

A database of 5203 patients treated with RAI for benign thyroid disease was established. The patients were at least 18 years of age, treated with RAI between 1975 and 2008 for either hyperthyroidism or symptomatic goitre at three university hospitals in Copenhagen, Denmark. Date of birth, date of first RAI treatment, administrated dose of ${ }^{131}$ I, thyroid status (hyperthyroid or euthyroid) and scintigraphic and/or ultrasound characteristics (i.e. diffuse or multi nodular) were registered throughout the years. The patients were categorized as euthyroid if they had a TSH $>0.1$, never been hyperthyroid and not on any thyroid medication, and were classified as hyperthyroid if they had a biochemically confirmed diagnosis of overt hyperthyroidism or if they had a TSH below $0.1 \mathrm{mU} / 1$ measured at least twice while not on any thyroid medication.

The patients, with unknown thyroid status $(n=44)$, receiving a total administrated dose of ${ }^{131}$ I over $2000 \mathrm{MBq}$ $(n=60)$, who had an invalid identification number $(n=68)$, and patients with ambiguous data $(n=2)$ were excluded (see Fig. 1). The characteristics of the excluded subjects were no different than the rest of the patients. The aimed target dose to the thyroid was between 90 and $150 \mathrm{~Gy}$, depending on the characteristics of the thyroid. Exclusion of patients receiving more than $2000 \mathrm{MBq}$ was done to mimic a normal treatment of benign thyroid disease in Denmark. In this study, the standard treatment regime is one dose of $200-600 \mathrm{MBq}$ (with a maximum of $600 \mathrm{MBq}$ according to outpatient radiation protection regimes). Some patients will however need a second dose (and a few even more) due to treatment failure.

Resident citizens in Denmark have a unique identification number in the Danish Civil Registration System linking hospital contacts through the Danish National Patient Registry (13). All remaining cases with a valid identification number were included $(n=5135)$. The patients were matched on age and sex with four randomly selected controls from the Danish background population through the Danish Civil Registration System ( $n=20$ 540). All controls had to be without known thyroid diagnosis in the Danish National Patient Registry and alive on their

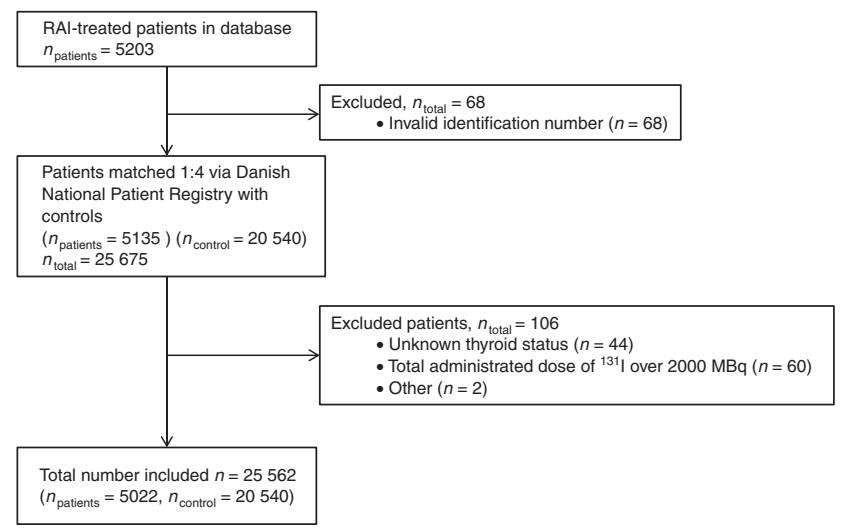

Figure 1

Flowchart of inclusion. 
index patient's day of treatment. Four controls per case were chosen to optimize the sample size and the statistical power of the study.

The study and the database were both approved by the local data safety section (GLO-2012-25) and by the national data safety section (FSEID-00000388).

\section{Outcome}

The primary outcome was defined as a difference in HR of the combined endpoint of hospitalization from cerebrovascular events among controls, hyperthyroid and euthyroid. Cerebrovascular events were defined as stroke (not haemorrhagic stroke in ICD10), transient ischaemic attack (TIA) and amaurosis fugax. Secondary outcomes were defined as the difference between all RAI-treated patients and controls, and between hyperthyroid and euthyroid when compared directly. We did not perform a dose-response analysis between administrated dose of ${ }^{131} \mathrm{I}$ and main outcome as there is no simple relationship between administrated dose and absorbed dose to the carotid arteries (2).

All cases and controls were followed from day of RAI until cerebrovascular event, death, emigration, maximum of 20 years follow-up or end of follow-up period on the 10th March 2013, whichever occurred first.

Information on the outcomes (and comorbidity) was obtained through National Patient Registry by use of identification numbers and prespecified ICD codes (see Table 1). In the National Patient Registry, an individual's hospital discharges are diagnosed with a primary diagnosis is of ICD8 (up until 1994) or ICD10. The diagnoses of cerebrovascular diseases have been validated in the National Patient Registry and have a relatively moderateto-high positive predictive value of about $80 \%$ for stroke and $65 \%$ for TIA (14). Data on date of death were obtained through death certificates in Danish National Death Register and Danish Civil Registration.

\section{Comorbidity}

Comorbidity, such as diabetes, rheumatic disease and heart disease, were identified through the National Patient Registry (see Table 1). Components of the Charlson comorbidity score (CCS) until inclusion date was retrieved, and an individual comorbidity score was created. The CCS has been validated to some extent for prediction of outcome after stroke (15). The ICD coding of CCS in the National Patient Registry has been validated (16).

Information on lipid profile, family history and social economical status was not available for neither patients nor controls. Furthermore, we do not have data on smoking status on neither the hyperthyroid nor the control group. However, we have smoking data for $\sim 20 \%$ ( $n=193$ ) of the euthyroid patients included in this study from another ongoing study (J L la Cour, U B Andersen, C $\mathrm{H}$ Sorensen, B Nygaard and L T Jensen; unpublished observations, registration number at the Ethical Committee in the Capital Region of Denmark: H-12012-120). In this euthyroid subgroup, the number of active smokers is comparable with the Danish

Table 1 ICD codes used in study.

\begin{tabular}{l} 
Disorder \\
\hline Main outcome \\
Stroke \\
Transient ischaemic attack \\
Amaurosis fugax \\
CCS \\
Cerebral vascular disease \\
Peripheral vascular disease \\
Myocardial infarction \\
Congestive heart failure \\
Malignancy \\
Diabetes \\
Pulmonary disease \\
Dementia \\
Mild liver disease \\
Hemiplegia \\
Renal disease \\
Rheumatic disease \\
Ulcer \\
AIDS
\end{tabular}

\begin{tabular}{l}
\hline ICD8 \\
\hline $432-434 ; 436-438$ \\
435 \\
377.02 \\
$430-438$ \\
$440-445$ \\
410 \\
$427.09 ; 428.99 ; 782.49$ \\
$140-209$ \\
$249-250$ \\
$490-492 ; 515.99-518$ \\
$290 ; 293.09$ \\
$456-457 ; 570-571 ; 573.1 ; 573.4$ \\
344 \\
$403-404 ; 582-584 ; 590 ; 593.19 ; 753 ; 792$ \\
$135.99 ; 446 ; 712 ; 714.95-716$ \\
$530.91 ; 530.98 ; 531-535$ \\
079.83
\end{tabular}

\section{ICD10}

163-68

G54-59

H34; G453

I60-69; G45-46

I70-74; 1771; R02

$121-23 ; 125$

I42-43; I50; I51.7; I110; I13.0

C00-97

E10-14

J41-68; J82-83; J84. 1; J98.2; J98.3

F00-03; F05.1; G31

B15.0; B16; K70-74; K76; 185

G81-82

I12-13; N0-5; N7; N11; N14; N17-19; Q64

M05-06; M08-09; M30-36; D86

K22; K25-29

B21-24 
background population (19\% vs 21\%; Statistics Denmark, http://www.dst.dk/da/Statistik/emner/de-kommunaleserviceindikatorer/sundhed, accesed 24th October 2014).

\section{Statistical analyses}

All baseline data are presented as percentages for categorical data and for continuous as means \pm s. $D$. when data followed a normal distribution or medians and interquartile range (IQR) when they did not.

Overall risk of being hospitalized with a cerebrovascular event was illustrated using Kaplan-Meier failure plot and log-rank testing for the comparison between groups. Competing risk regression with death as the competing risk was used to test the risk of hospitalization due to cerebrovascular events among hyperthyroid, euthyroid and controls adjusting for risk factors of age and sex in a basic model. In the final model, CCS $(\geq 2)$, AF and previous cerebrovascular event as categorical variables (yes/no) were included. Model control was done by plotting proportional hazards.

All statistical analyses were performed with SAS Statistical Software version 9.3 (SAS Institute, Cary, NC, USA) or Stata Software version 11 (StataCorp., College Station, TX, USA). A two-sided $P$ value $<0.05$ was considered to be statistically significant.

Sensitivity analyses $\triangleright$ A separate test was done for stroke alone (not including amaurosis fugax and TIA) in the full competing risks model, to ensure that the most important endpoint was consistent with the test of the combined endpoint. To see whether there was more systemic atherosclerosis in the RAI-treated groups than in the control group, we tested the risk of developing acute myocardial infarction (ICD8: 410 and ICD10: I21-22) after inclusion by a log-rank test and by Mantel-Haenszel analysis adjusted for age, sex, prior ischaemic heart disease, AF and CCS (data did not fit the assumptions of competing risk).

Furthermore, we tested whether treatment year influenced the HR by splitting on treatment year as different treatment regimens may have been applied. In addition, we tested whether diffuse or multi-nodular thyroid disease influenced the risk of cerebrovascular events.

\section{Results}

A total of 25562 subjects, 14.3\% men, were followed with a mean follow-up time of 11.5 years $( \pm 6.1)$ and a total number of person years at risk of 294230 . The mean age at inclusion was 61 years $( \pm 14.2)$, and the total number of events were 3228. Before or on the day of inclusion, 468 (1.8\%) subjects had been diagnosed with AF, and 1305 (18.6\%) had a CCS of two or more. Both the RAI-treated groups had more comorbidity than the control group, and the euthyroid group was younger than the other groups (see Table 2).

When testing whether there was a difference between the control group and the whole RAI-treated group (including both the hyperthyroid and the euthyroid), we found a significant difference using Kaplan-Meier failure plot and log-rank testing $(P<0.001)$. In the basic competing risk analysis model (adjusting for age and sex), the RAI-treated group had an increased risk of

Table 2 Characteristics of the included patients and controls. Number of events denotes the absolute number of hospitalizations from cerebrovascular disease (stroke, transient ischaemic attack and amaurosis fugax) for each group. Charlson comorbidity score (CCS) is an individual score of comorbidity using 19 different diseases giving them a point from 1 to 6 and from that generate one score from 0 to 6 per individual. Atrial fibrillation (AF) refers to persons with the disease registered before or at time of treatment. Total dose of ${ }^{131} \mathrm{I}$ refers to the individual patients accumulated dose of ${ }^{131}$ I given at the three included hospitals from 1976 to 2008 .

\begin{tabular}{|c|c|c|c|c|}
\hline & Control $(n=20540)$ & Hyperthyroid $(n=4000)$ & Euthyroid $(n=1022)$ & Total $(n=25562)$ \\
\hline Person time at risk (years) & 238367 & 43790 & 12073 & $294230^{\dagger}$ \\
\hline $\begin{array}{l}\text { Subjects with a cerebrovascular event } \\
\text { after inclusion, } n(\%)\end{array}$ & $2508(12.2 \%)$ & $584(14.6 \%)$ & $136(13.3 \%)$ & $3228(12.6 \%)^{\dagger}$ \\
\hline Age at inclusion, mean years (s.D.) & $61.0(14.2)$ & $61.9(14.3)$ & $57.8(13.3)$ & $61.0(14.2) *$ \\
\hline Sex (male), \% & 14.3 & 14.7 & 12.1 & 14.3 \\
\hline $\mathrm{CCS} \geq 2(\%)$ & 4.7 & 7.0 & 6.3 & $5.1^{\dagger}$ \\
\hline$A F(\%)$ & 1.5 & 4.9 & 3.4 & $1.8^{\dagger}$ \\
\hline Total dose of ${ }^{131} \mathrm{I}$, median MBq (IQR) & - & $400(304)$ & 407 (303) & $400(304) *$ \\
\hline $\begin{array}{l}\text { Subjects with a cerebrovascular event } \\
\text { before inclusion date (\%) }\end{array}$ & 3.8 & 4.8 & 4.0 & $4.0 *$ \\
\hline
\end{tabular}


Table 3 The results from the main competing risk analyses of risk of hospitalization from cerebrovascular events (combined endpoint of stroke, transient ischemic attack and amaurosis fugax) after therapy with radioactive iodine for benign thyroid disorders. Maximum follow up of 20 years. Death was the competing risk in all analyses.

\begin{tabular}{|c|c|c|}
\hline \multirow[b]{2}{*}{ Main results } & \multicolumn{2}{|c|}{ Hazard ratio } \\
\hline & In a model adjusted for age and sex $(95 \% \mathrm{Cl})$ & $\begin{array}{l}\text { In a model adjusted for age, sex, } \mathrm{CCS}, \mathrm{AF} \text { and } \\
\text { previous cerebrovascular event }(95 \% \mathrm{Cl})\end{array}$ \\
\hline All RAl treated vs control & $1.21(1.11-1.31)$ & $1.18(1.09-1.29) * *$ \\
\hline \multicolumn{3}{|l|}{ Patient groups compared with controls } \\
\hline Hyperthyroid & $1.20(1.1-1.32)$ & $1.18(1.07-1.29) * *$ \\
\hline Euthyroid & $1.23(1.04-1.46)$ & $1.22(1.02-1.44) *$ \\
\hline Hyperthyroid vs euthyroid & $0.97(0.8-1.17)$ & $0.96(0.8-1.16)$ \\
\hline
\end{tabular}

${ }^{*} P<0.01 ; * * P<0.001$

cerebrovascular events compared with controls of hazard ratio (HR) 1.21 (95\% CI 1.11-1.31; see Table 3). Adding CCS, AF and previous cerebrovascular event to the model only affected the estimates slightly, HR 1.18 (95\% CI 1.091.29; see Table 3).

When testing whether the risk of cerebrovascular events was explained by thyroid status, we split the patient group into hyperthyroid and euthyroid and found a difference between the thyroid healthy control group and both the RAI-treated groups (hyperthyroid and euthyroid; log-rank testing, $P<0.0001)$. In the basic competing risk model, the hyperthyroid as well as the euthyroid had an increased risk of cerebrovascular events compared with the control group, with an HR 1.20 (95\% CI 1.11-1.32) and HR 1.23 (95\% CI 1.04-1.46) respectively (see Table 3 ). It only changed the estimates slightly when we adjusted for CCS, AF and previous cerebrovascular event, HR 1.17 (95\% CI 1.07-1.29) for hyperthyroid group and HR 1.21 (95\% CI 1.02-1.44) for euthyroid group (see Table 3 and Fig. 2). When testing hyperthyroid and euthyroid against each other, we find no difference (see Table 3).

\section{Sensitivity analyses}

The separate analysis of stroke alone as outcome did not differ from the main analysis (data not shown). In terms of acute myocardial infarction, a difference was seen between the control and the hyperthyroid groups $(P<0.0001$ for logrank; relative risk $(\mathrm{RR})=1.12(95 \% \mathrm{CI} 1.01-1.25) ; P=0.034$ for Mantel-Haenszel), not between the control group and euthyroid group $(P=0.29$ for log-rank; $\mathrm{RR}=1.14$ (95\% CI $0.93-1.4) ; P=0.2$ ). No difference was observed in the analysis on treatment year (data not shown) or when comparing diffuse and multi-nodular thyroid disease (data not shown).

\section{Discussion}

In this cohort study, we report an increased risk of cerebrovascular events after RAI in 4000 hyperthyroid and 1022 euthyroid patients compared with 20540 controls followed for a total of 294230 follow-up years. This is the first study evaluating the possible risk of RAIinduced cerebrovascular events differentiating between euthyroid patients and hyperthyroid patients.

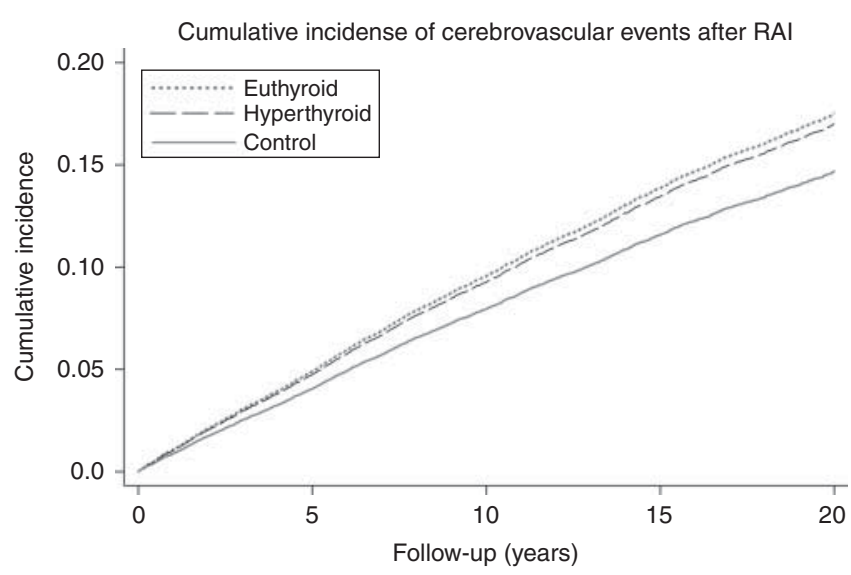

\section{Figure 2}

Cumulative incidence of cerebrovascular events. Cumulative incidence of hospitalization from the combined endpoint cerebrovascular event (stroke, transient ischaemic attack and amaurosis fugax) after therapy with radioactive iodine (RAI) in a competing risk model adjusted for age, sex, atrial fibrillation, Charlson comorbidity score (CCS) and previous cerebrovascular event. Death is the competing risk. Patients (both hyper- and euthyroid) and controls enter at the day of the patients' therapy with RAl. Figure illustrates the difference among the hyperthyroid and euthyroid groups compared with the control group. 
The main finding is that euthyroid patients treated with RAI have increased risk of cerebrovascular events (stroke, amaurosis fugax and TIA) with a HR 1.22 (1.021.44) compared with the control group in our fully adjusted model. The risk is comparable with the established risk of cerebrovascular events and mortality among RAI-treated hyperthyroid (HR 1.18; 95\% CI 1.07-1.29). If hyperthyroidism was the only explaining factor of the excess risk of cerebrovascular events, we would have expected to find a difference between euthyroid and hyperthyroid patients. None of the sensitivity analyses changed the confidence in our results. In fact, that the mechanism behind the increased risk of cerebrovascular events might be a local radiation effect, not a systemic reaction to RAI or difference in the general atherosclerosis burden between the groups, is strengthened by the information that the euthyroid group and the control group have similar risks of developing acute myocardial infarction. Our results therefore indicate that other factors than hyperthyroidism might explain the increased risk of cerebrovascular events in the RAI-treated group. As discussed, one of these factors could be radiation-induced atherosclerosis in the carotid arteries.

In order for RAI to induce atherosclerosis and thereby increase the risk of stroke, the carotid arteries have to be exposed during treatment. Through computer simulation, we have recently investigated whether this is in fact the case (2). We found that the carotid arteries are exposed to an average committed dose of $4-50 \mathrm{~Gy} / \mathrm{GBq}{ }^{131} \mathrm{I}$ orally administrated with a dose rate of $4.2 \mathrm{~Gy} / \mathrm{GBq}$ in a standardized situation (2). This amount of energy is well above the estimated lower limit of $2 \mathrm{~Gy}$ to induce an inflammatory response and initiate or accelerate atherosclerosis (17). This means that radiation-induced atherosclerosis could be an explanatory factor. That radiation-induced atherosclerosis could perhaps evolve after RAI is shown a study by Surucu et al. (18) who measure carotid intima media thickness (CIMT; a fairly validated surrogate for early atherosclerosis (19)) in hyperthyroid patients treated with RAI. They report a lasting increase in CIMT after RAI. However, the study has several limitations. First and foremost is that they do not account for changes in cholesterol and TSH, which are known to affect CIMT (20). However, the result does fall in line with our studies and studies comparable with our current epidemiological study.

In UK, Franklyn et al. (3) followed 7209 hyperthyroid patients treated with RAI and find a standardized mortality ratio of 1:4 (95\% CI 1.2-1.5) for mortality from cerebrovascular events. Metso et al. $(4,21)$ followed RAI-treated hyperthyroid and controls in Finland and find an increased risk of mortality and morbidity from hospitalization due to ischaemic and embolic cerebrovascular events $(\mathrm{RR}=1.31$ (1.14-1.56) in (4), and 1.40 (1.16-1.69) in (21)). The finding of similar risk sizes across three RAI-treated hyperthyroid populations indicates a correlation. Still the results do not explain whether it is hyperthyroidism, RAI therapy or the combination that increases the risk.

Trying to help answer the question of hyperthyroidism vs treatment, Sheu et al. (7) found an increased risk of stroke of $1.44(1.02-2.12)$ among young Taiwanese patients on thyroid medication. However, in Finland Ryodi et al. (8) did a register study on surgically treated hyperthyroid patients and found an increased risk of hospitalization from all-cause cardiovascular events, but not from cerebrovascular events $(P=0.057)$. This strengthens our fear of RAI playing a role in the increased risk of cerebrovascular events after RAI. However, the results of these studies illustrate that the debate on treatment vs disease remains controversial.

\section{Strengths and limitations}

We did not have access to the actual TSH values. A cut off of TSH of 0.1 was used during categorization throughout the years. This leads to some of the mild subclinical hyperthyroid (defined as TSH below normal range but above 0.1 and normal $\mathrm{T}_{4}$ and free $\mathrm{T}_{3}$ ) being included in the euthyroid group. This could lead to an overestimation of the risk of cerebrovascular events in this group, as these individuals have an increased risk of AF (22). However, whether subclinical hyperthyroidism leads to an increased risk of stroke is a subject of debate. A cross-sectional study found an increased risk of stroke (23); however, a recent metaanalysis by Chaker et al. (24) and a recent very large study not included in the meta analysis by Selmer et al. (10) found no evidence of increased risk in subjects with mild subclinical hyperthyroidism. Therefore, whether this classification of TSH $<0.1$ leads to an overestimation of the risk of cerebrovascular events in our study due to mild subclinical hyperthyroidism is uncertain.

No post-RAI TSH levels were available, which is a limitation because there is a connection between thyroid function after RAI and survival (25). The study by Boelaert et al. finds that patients who develop a need for thyroid hormone substitution after RAI survive the longest. However, the connection between post-RAI TSH levels and cerebrovascular events is unknown, and it is therefore difficult to estimate how knowing these values would affect our results. 
There may be a difference in comorbidity not requiring hospitalization between the groups, which we do not find, because we only include hospitalizations. Furthermore, some minor TIAs might be only treated at the general practice and therefore not included in our data. This would lead to an underestimation of the risk of cerebrovascular events in total. However, there is no reason to believe that more people in the control group would have one of these minor TIAs than patients in the two other groups. If anything, following our theory of radiation-induced atherosclerosis it would be the other way around. This would lead to an underestimation of the risk. However, given that we have more than 3000 events, the effects of these unrecorded TIAs are not likely to affect our results.

The problem of residual confounding is always there in the register-based design. We cannot account for differences in the socioeconomic status, traditional cardiovascular risk factors (i.e. cholesterol and obesity), and smoking status between the patients and the controls. As smoking and socioeconomical status are factors predisposing thyroid illness and stroke, these are expected to play a role in our findings, especially among the hyperthyroid patients (26). However, in our subpopulation of euthyroid patients the frequency of smokers was comparable with the background population. To our knowledge, there are no studies indicating increased prevalence of smokers among euthyroid treated with RAI compared with the general population. Therefore, the increased risk of cerebrovascular events in this group is not likely solely to be attributed to residual confounding.

A control group treated with a different modality, i.e. medication or surgery, would have made an even more convincing argument. These patients are unfortunately not included in our database, and even if they were, there would still be the problem with selection bias in a retrospective design.

The major strength of our current study is that we have a large cohort of both hyperthyroid and euthyroid patients treated with RAI followed in a highly validated register (14).

\section{Conclusion}

It is known that ionizing radiation can induce atherosclerotic events. During therapy, RAI exposes the carotid arteries to radiation. Therefore, RAI therapy might increase the risk of cerebrovascular atherosclerotic events. In this study, we report an increased risk of cerebrovascular events among hyperthyroid as well as euthyroid treated with RAI for benign thyroid disorders. The finding of increased risk of the same magnitude in these two groups indicates that it is not solely hyperthyroidism that explains the previously noticed increased risk of cerebrovascular events after RAI. This study raises the possibility that therapy with RAI might play the most important role. Therefore, this information should perhaps be taken into consideration when advising patients with benign thyroid disease in the choice of treatment modality.

\section{Declaration of interest}

The authors declare that there is no conflict of interest that could be perceived as prejudicing the impartiality of the research reported.

\section{Funding}

This work was supported by unspecific grants from Agnes and Knut Mørk Foundation (no grant number given); and Danish Thyroid Foundation (Thyreoidea Landspatientforeningen) (grant number 1301652).

\section{References}

1 Darby SC, Ewertz M, McGale P, Bennet AM, Blom-Goldman U, Bronnum D, Correa C, Cutter D, Gagliardi G, Gigante B et al. Risk of ischemic heart disease in women after radiotherapy for breast cancer. New England Journal of Medicine 2013368 987-998. (doi:10.1056/ NEJMoa1209825)

2 la Cour JL, Hedemann-Jensen P, Sogaard-Hansen J, Nygaard B \& Jensen LT. Modeling the absorbed dose to the common carotid arteries following radioiodine treatment of benign thyroid disease. Annals of Nuclear Medicine 201327 862-866. (doi:10.1007/s12149-013-0756-y)

3 Franklyn JA, Maisonneuve P, Sheppard MC, Betteridge J \& Boyle P. Mortality after the treatment of hyperthyroidism with radioactive iodine. New England Journal of Medicine 1998338 712-718. (doi:10.1056/NEJM199803123381103)

4 Metso S, Auvinen A, Salmi J, Huhtala H \& Jaatinen P. Increased longterm cardiovascular morbidity among patients treated with radioactive iodine for hyperthyroidism. Clinical Endocrinology 200868 450-457. (doi:10.1111/j.1365-2265.2007.03064.x)

5 Stuijver DJ, van Zaane ZB, Romualdi E, Brandjes DP, Gerdes VE \& Squizzato A. The effect of hyperthyroidism on procoagulant, anticoagulant and fibrinolytic factors: a systematic review and meta-analysis. Thrombosis and Haemostasis 2012108 1077-1088. (doi:10.1160/TH12-07-0496)

6 Biondi B \& Kahaly GJ. Cardiovascular involvement in patients with different causes of hyperthyroidism. Nature Reviews. Endocrinology 2010 6 431-443. (doi:10.1038/nrendo.2010.105)

7 Sheu JJ, Kang JH, Lin HC \& Lin HC. Hyperthyroidism and risk of ischemic stroke in young adults: a 5-year follow-up study. Stroke 2010 41 961-966. (doi:10.1161/STROKEAHA.109.577742)

8 Ryodi E, Salmi J, Jaatinen P, Huhtala H, Saaristo R, Valimaki M, Auvinen A \& Metso S. Cardiovascular morbidity and mortality in surgically treated hyperthyroidism - a nation-wide cohort study with a long-term follow-up. Clinical Endocrinology 201480 743-750. (doi:10.1111/cen.12359)

9 Parle JV, Maisonneuve P, Sheppard MC, Boyle P \& Franklyn JA. Prediction of all-cause and cardiovascular mortality in elderly people 
from one low serum thyrotropin result: a 10-year cohort study. Lancet 2001358 861-865. (doi:10.1016/S0140-6736(01)06067-6)

10 Selmer C, Olesen JB, Hansen ML, von Kappelgaard LM, Madsen JC, Hansen PR, Pedersen OD, Faber J, Torp-Pedersen C \& Gislason GH. Subclinical and overt thyroid dysfunction and risk of all-cause mortality and cardiovascular events: a large population study. Journal of Clinical Endocrinology and Metabolism 201499 2372-2382. (doi:10.1210/ jc.2013-4184)

11 Flynn RW, Macdonald TM, Jung RT, Morris AD \& Leese GP. Mortality and vascular outcomes in patients treated for thyroid dysfunction. Journal of Clinical Endocrinology and Metabolism 200691 2159-2164. (doi:10.1210/jc.2005-1833)

12 Hoffman DA, McConahey WM, Diamond EL \& Kurland LT. Mortality in women treated for hyperthyroidism. American Journal of Epidemiology $1982115243-254$.

13 Lynge E, Sandegaard JL \& Rebolj M. The Danish National Patient Register. Scandinavian Journal of Public Health 201139 30-33. (doi:10.1177/1403494811401482)

14 Krarup LH, Boysen G, Janjua H, Prescott E \& Truelsen T. Validity of stroke diagnoses in a National Register of Patients. Neuroepidemiology 200728 150-154. (doi:10.1159/000102143)

15 Jimenez Caballero PE, Lopez EF, Portilla Cuenca JC, Ramirez Moreno JM, Pedrera Zamorano JD \& Casado NI. Charlson comorbidity index in ischemic stroke and intracerebral hemorrhage as predictor of mortality and functional outcome after 6 months. Journal of Stroke and Cerebrovascular Diseases 201322 e214-e218. (doi:10.1016/j.jstrokecerebrovasdis. 2012.11.014)

16 Thygesen SK, Christiansen CF, Christensen S, Lash TL \& Sorensen HT. The predictive value of ICD-10 diagnostic coding used to assess Charlson comorbidity index conditions in the population-based Danish National Registry of Patients. BMC Medical Research Methodology 201111 83. (doi:10.1186/1471-2288-11-83)

17 Stewart FA. Mechanisms and dose-response relationships for radiationinduced cardiovascular disease. Annals of the ICRP 201241 72-79. (doi:10.1016/j.icrp.2012.06.031)

18 Surucu E, Bekis R, Sengoz T, Demir Y, Celik AO, Orbay O, Birlik B, Ozdogan O, Igci E \& Durak H. The effect of radioiodine on the intima media thickness of the carotid artery. Molecular Imaging and Radionuclide Therapy 201322 85-89. (doi:10.4274/Mirt.24119)

19 van den Oord SC, Sijbrands EJ, ten Kate GL, van Klaveren D, van Domburg RT, van der Steen AF \& Schinkel AF. Carotid intima-media thickness for cardiovascular risk assessment: systematic review and meta-analysis. Atherosclerosis 2013228 1-11. (doi:10.1016/j.atherosclerosis.2013.01.025)

20 Volzke H, Robinson DM, Schminke U, Ludemann J, Rettig R, Felix SB, Kessler C, John U \& Meng W. Thyroid function and carotid wall thickness. Journal of Clinical Endocrinology and Metabolism 200489 2145-2149. (doi:10.1210/jc.2003-031028)

21 Metso S, Jaatinen P, Huhtala H, Auvinen A, Oksala H \& Salmi J. Increased cardiovascular and cancer mortality after radioiodine treatment for hyperthyroidism. Journal of Clinical Endocrinology and Metabolism 200792 2190-2196. (doi:10.1210/jc.2006-2321)

22 Selmer C, Olesen JB, Hansen ML, Lindhardsen J, Olsen AM, Madsen JC, Faber J, Hansen PR, Pedersen OD, Torp-Pedersen C et al. The spectrum of thyroid disease and risk of new onset atrial fibrillation: a large population cohort study. BMJ 2012345 e7895. (doi:10.1136/bmj.e7895)

23 Dorr M, Empen K, Robinson DM, Wallaschofski H, Felix SB \& Volzke H. The association of thyroid function with carotid artery plaque burden and strokes in a population-based sample from a previously iodinedeficient area. European Journal of Endocrinology 2008159 145-152. (doi:10.1530/EJE-08-0140)

24 Chaker L, Baumgartner C, Ikram MA, Dehghan A, Medici M, Visser WE, Hofman A, Rodondi N, Peeters RP \& Franco OH. Subclinical thyroid dysfunction and the risk of stroke: a systematic review and metaanalysis. European Journal of Epidemiology 201429 791-800. (doi:10.1007/s10654-014-9946-8)

25 Boelaert K, Maisonneuve P, Torlinska B \& Franklyn JA. Comparison of mortality in hyperthyroidism during periods of treatment with thionamides and after radioiodine. Journal of Clinical Endocrinology and Metabolism 201398 1869-1882. (doi:10.1210/jc.2012-3459)

26 Knudsen N, Bulow I, Laurberg P, Ovesen L, Perrild H \& Jorgensen T. Low socio-economic status and familial occurrence of goitre are associated with a high prevalence of goitre. European Journal of Epidemiology 2003 18 175-181. (doi:10.1023/A:1023001400945)

Received 12 December 2014

Revised version received 25 February 2015

Accepted 19 March 2015 